\title{
CLINICAL STUDY OF PREOPERATIVE NECK MASSES WITH COMPUTED TOMOGRAPHY (CT)/ MAGNETIC RESONANCE IMAGING (MRI) FINDINGS AND FINE NEEDLE ASPIRATION CYTOLOGY (FNAC) FOR APPROPRIATE SURGICAL MANAGEMENT
}

\author{
Srirangaprasad K1, George K. George², Pruthvi Raj $S^{3}$ \\ 1 Professor, Department of ENT-Head and Neck Surgery, Rajarajeswari Medical College and Hospital, Bangalore, Karnataka. \\ ${ }^{2}$ Senior Resident, Department of ENT-Head and Neck Surgery, Rajarajeswari Medical College and Hospital, Bangalore, Karnataka. \\ ${ }_{3}^{3}$ Postgraduate Student, Department of ENT-Head and Neck Surgery, Rajarajeswari Medical College and Hospital, Bangalore, \\ Karnataka.
}

\section{BACKGROUND}

ABSTRACT

The head and neck are the regions that present both high anatomical and functional difficulties, making the precise diagnosis a challenging task. Meticulous clinical history and physical examination may suggest the clinical diagnosis. Imaging is increasingly performed to assess the anatomical extent of involvement before any form of treatment and also helps in the differential diagnosis. Fine needle aspiration cytology (FNAC) is gold standard for diagnosis of any neck mass.

Aims and Objectives- The objective was to assess the accuracy of CT/ MRI scan and FNAC in prediction of neck masses, so that a suitable surgical neck dissection can be carried out.

\section{MATERIALS AND METHODS}

Data for the study was collected from patients presenting to Department of ENT at Rajarajeswari Medical College and Hospital, Bangalore with complaints of non-thyroidal swelling in neck region from 1st October 2013 - 30th September 2015. Patients consenting for the study and meeting the selection criteria were included in the study. FNAC and CT/ MRI of the swelling were done on an OPD basis for the patients.

\section{RESULTS}

A total of 96 patients presented in our OPD with complaints related to non-thyroidal neck swellings. CT/ MRI scan was done for all cases. 85 patients came for follow-up and they underwent FNAC. 11 were unsatisfactory aspirates consisting of blood or inadequate aspirate. Thus, a total of 74 cases were included in this study. Age group of the patients referred for aspirations ranged from 12 years to 73 years with mean age of 45.62 years. In this study, provisional diagnosis based on CT/ MRI was correlated with FNAC. Correlation was present in the $61.5 \%$ of non-thyroidal neck swelling.

\section{CONCLUSION}

Contrast enhanced CT scans has improved the localisation and characterisation of neck lesions, but FNAC remains the gold standard for making a histological diagnosis. Hence, a combination of CT/ MRI and FNAC will give comprehensive information regarding the nature of lesion and its extent, which is absolutely necessary for diagnosis and staging of the disease for planning the treatment modality to be adapted.

\section{KEYWORDS}

Neck Swelling, Fine Needle Aspiration Cytology, CT/ MRI.

HOW TO CITE THIS ARTICLE: Srirangaprasad K, George GK, Raj PS. Clinical study of preoperative neck masses with computed tomography (CT)/ magnetic resonance imaging (MRI) findings and fine needle aspiration cytology (FNAC) for appropriate surgical management. J. Evolution Med. Dent. Sci. 2018;7(18):2261-2265, DOI: 10.14260/jemds/2018/509

\section{BACKGROUND}

The head and neck are the regions that present both high anatomical and functional difficulties, making the precise diagnosis and staging of regional tumours a challenging task. A good clinical history, physical examination may suggest the clinical diagnosis. Many neoplasms are detected at clinical examination, but imaging techniques are also necessary for characterisation of biological aggressiveness and staging.

'Financial or Other Competing Interest': None.

Submission 17-03-2018, Peer Review 12-04-2018,

Acceptance 18-04-2018, Published 30-04-2018.

Corresponding Author:

Dr. Srirangaprasad $K$,

C/o. House No. 424, $7^{\text {th }}$ Cross,

$1^{\text {st }}$ Block, Jayanagar,

Bangalore-560011,

Karnataka.

E-mail: ranganitha@yahoo.co.in

DOI: $10.14260 / \mathrm{jemds} / 2018 / 509$
Morphological imaging techniques such as CT and MRI provide anatomical information that is not always enough for the evaluation of biological characteristics of tumours in the head and neck areas.

Magnetic resonance images reflect tissue biochemistry and are particularly influenced by the presence of protons within the tissues. T1-weighted images carry a great deal of spatial resolution with excellent depiction of detailed anatomy. T2-weighted images are better at highlighting abnormal tissues. The ability of MRI therefore is to show abnormal tumour tissue as high signal and normal tissue as low signal in an image which creates improved contrast resolution when compared to CT. MRI will not be suitable for all patients with head and neck lesions. Although, CT is superior to MRI in assessment of cortical bone, MRI has superior soft tissue contrast compared to CT and allows true multiplanar imaging. ${ }^{1}$

Diffusion weighted imaging (DWI) have been largely used to make early diagnosis and management of ischaemic 
pathology in the brain and later in the evaluation of white matter disorders and brain tumours. Recent technological advancements have allowed its use in the differentiation between malignant and benign lesions outside the brain. DWI may be measured by means of apparent diffusion coefficient (ADC). Areas of decreased ADC values within tumours of different regions correlate with areas of increased cellularity within tumours. Therefore, DWI has been observed as a powerful imaging biomarker of cancer. ${ }^{1}$

Fine needle cytology is now a widely accepted and successful diagnostic technique in most of the neck swellings, especially in oncology.

FNAC is of particular relevance in the head and neck area because of easy accessibility of the target site, excellent patient compliance, minimally invasive nature of the procedure and helping to avoid surgery in non-neoplastic lesions, inflammatory conditions and also some tumours and is a simple, cost effective, readily repeated and quick to perform procedure in the outpatient department with excellent patient compliance. ${ }^{2}$

The accuracy of cytological diagnosis depends on the expertise of the cytopathologist, technician, the site of lesion, the adequacy of sample and the sampling method. ${ }^{3}$

The objective of the study was to assess the accuracy of CT/ MRI scan and FNAC in prediction of neck masses, so that a suitable surgical management can be done.

\section{MATERIALS AND METHODS}

It was an observational study and data was collected from patients presenting to Department of ENT, Head and Neck Surgery at Raja Rajeswari Medical College and Hospital, Bangalore with complaints of non-thyroidal swelling in neck region from $1^{\text {st }}$ October 2013 - 30th September 2015. An informed written consent was taken from the patient and cases meeting the selection criteria were included in the study. The inclusion criteria were the patients with nonthyroidal neck swelling and the patients with thyroid swelling, all patients with co-morbidities and unfit for surgery, all patients who did not give consent were excluded. FNAC is considered the gold standard diagnostic test in the evaluation of a swelling of thyroid nodule. By using CT/ MRI we accessed the specificity and sensitivity of FNAC in detecting thyroid swelling.

FNAC and CT/ MRI of the swelling were done on an OPD basis for all consenting patients presenting with neck swelling.

The materials used were $10 \mathrm{~mL}$ disposable plastic syringe, 1.5 inch 23-G disposable needle, micro slides $(7.5 \times$ $2.5 \mathrm{~cm}$ size), fixatives (95\% Isopropyl alcohol and ether and 2-5 drops of acetaldehyde), spirit swab to sterilise the skin and stains [Haematoxylin and Eosin stain ( $\mathrm{H}$ and E), Giemsa stain, Papanicolaou stain (Rarely)] and local anaesthesia was not used in any patient.

All the patients were clinically examined in detail and a careful palpation of the swelling was done to judge precisely the location for aspiration. After explanation about the procedure to the patient, aspiration was done with the patient in supine or sitting position with extended neck, so as to make the swelling appear prominent. The 1.5-inch 23-G disposable needle was used for aspiration. The needle is attached to a $10 \mathrm{~mL}$ disposable syringe and inserted into the swelling in a direction perpendicular to the anterior surface of neck. The opposite hand fixes the swelling as the needle is inserted. When the needle is in place, the opposite hand holds the syringe. The hand that inserted the needle withdraws the plunger to create suction. The opposite hand again fixes the swelling for the rest of the procedure. After the needle has been withdrawn from the swelling, it is detached from the syringe. A few $\mathrm{mL}$ of air is aspirated into the syringe, the needle is reattached and the air is expressed through the needle to expel the specimen onto a glass slide. The specimen is inspected to assess its suitability for cytologic evaluation. The specimen desired consists of a drop of red-orange fluid. 4,5

After the specimen has been expressed onto a glass slide, it is smeared. The frequently used technique of approximating the edge of a second slide at a 45-degree angle, permitting the fluid to flow along the edge of the top slide and then drawing the specimen out over the bottom slide produces thick uneven smears. A better technique is to place the top slide flat on the specimen and with the index finger press down and slide the top slide over the bottom slide. This produces a thin, evenly dispersed smear that is ideal for cytologic evaluation. 4,5

The smear was fixed immediately. The preferred stain was Haematoxylin and Eosin in our centre.

The most commonly used fixative is $95 \%$ ethyl alcohol, either by immersion of the smears or as a spray fixative. Spray fixations of smears is preferred by many laboratories, because it provides optimal cytomorphology with excellent nuclear detail, demonstration of differentiation and preservation of architectural features without red cell lysis or background staining. Haematoxylin and eosin staining was used for staining.

\section{The Cytological Smears were classified as-}

1. Inadequate- When the specimen contained insufficient material for cytological evaluation or had blood.

2. Benign- When the epithelial element showed uniformity of cell size, shape and nuclear structure.

3. Suspicious- Occasionally epithelial cells showed marked cellular changes suggestive of malignancy or when cells were abundant, but aggregated together in dense clumps precluding interpretation.

4. Malignant- When large number of non-cohesive epithelial cells showed marked variation in size, shape and nuclear structure, frequently with enlarged, irregular and multiple nuclei.

Data was entered in Microsoft Excel and analysed using SPSS (Statistical Package for Social Science, Ver. 10.0.5) package. The results were presented in number and percentage for dichotomous data in Table and Figure. FNAC is considered the gold standard diagnostic test in the evaluation of neck thyroid swelling.

\section{RESULTS}

A total of 96 patients presented in our OPD during the study period with complaints related to non-thyroidal neck swellings of varying sizes and time duration. CT/ MRI scan was done for all cases. Among these patients, 85 underwent FNAC. 11 were unsatisfactory aspirates consisting of blood or inadequate aspirate. The cases were operated, and specimen 
was available for histopathologic examination. Thus, a total of 74 cases were included in this study.

Age group of patients referred for aspirations ranged from 12 years to 73 years with mean age of 45.62 years. Majority of the patients were in the age group of $40-49$ years. Majority of them were males 46 cases (62.2 percent) and female constitutes about 28 cases ( 37.8 percent).

All the 74 patients presented with swelling in the neck region. One case presented with swelling behind left ear lobe extending into the neck region. Similarly, one case presented with swelling in front of left ear lobe. About 5 cases presented with midline swelling.

Out of 74 swelling, 58 neck swellings were non-tender and tenderness was present in 16 cases. Among 74 cases 35 cases presented as firm swelling, 34 cases were soft, and 5 cases were hard.

\section{Clinical Diagnosis}

Most of the swellings were diagnosed clinically as lipoma in about 19 cases, after that cervical lymph node enlargement most probably due to some secondaries in the neck region constitutes about 16 cases.

\section{Cytological Diagnosis}

Satisfactory cell sample was obtained in 85 patients, while in the remaining 11 cases aspiration was unsatisfactory consisting of blood and inadequate aspirate with satisfactory smear rate of $87.05 \%$. Most of the cases were cytologically diagnosed as squamous cell carcinoma about 26 cases, after that was lipoma about 19 cases.

\section{CT/ MRI Scan}

According to provisional diagnosis based on CT/ MRI, majority of the cases were lipoma which constitutes about 22 cases, 11 each of pleomorphic adenoma and parotid carcinoma, 10 cases were found to be cervical lymph node enlargement due to secondaries in the neck region.

All cases were surgically managed. 28 cases were excised. Excision and modified radical neck dissection were done in 26 cases. Superficial parotidectomy was done in 16 cases. Incision and drainage was done in 2 cases. One each case of resection and submandibular gland excision were done.

Provisional diagnosis based on CT/ MRI was correlated with Fine Needle Aspiration Cytology. Correlation was present in the $61.5 \%$ of non-thyroidal neck swelling. Of these, $38.4 \%$ of the swelling were benign and $23.07 \%$ of the swellings were malignant. About $75 \%$ of pleomorphic adenoma can be correlated with CT/ MRI. 66.7\% correlation was present in case of Warthin's tumour. $100 \%$ of adenoid cystic carcinoma, nerve sheath tumour and submandibular gland carcinoma were correlated with CT/ MRI report. 89.5\% of lipoma were correlated with CT/ MRI. $33.3 \%$ of sebaceous cyst were correlated with CT/ MRI findings. Other rare swelling which are correlated $100 \%$ were branchial cleft cyst, laryngocele, lymphangioma, parapharyngeal abscess and parotitis.

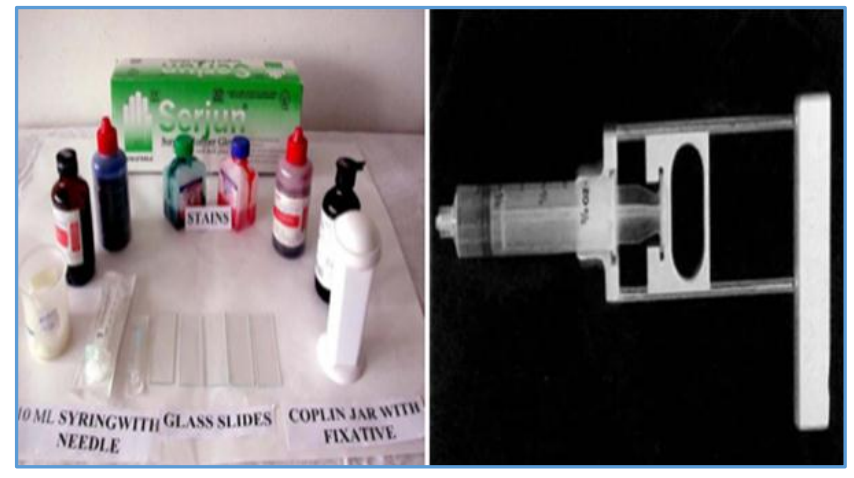

Fixator, Stains and Syringe Holder

\begin{tabular}{|c|c|c|}
\hline Symptoms & Frequency & Percent \\
\hline Swelling behind left ear lobe & 1 & 1.4 \\
\hline Swelling in front of left ear & 1 & 1.4 \\
\hline Swelling in front of neck & 5 & 6.8 \\
\hline Swelling on both sides of neck & 3 & 4.1 \\
\hline Swelling on left side of neck & 31 & 41.9 \\
\hline Swelling on right side of neck & 33 & 44.6 \\
\hline Total & $\mathbf{7 4}$ & $\mathbf{1 0 0 . 0}$ \\
\hline \multicolumn{2}{|c}{ Clinical Features } \\
\hline
\end{tabular}

\begin{tabular}{|c|c|c|}
\hline Clinical Diagnosis & Frequency & Percent \\
\hline Branchial cleft cyst & 1 & 1.4 \\
\hline Carcinoma larynx & 1 & 1.4 \\
\hline Cervical lymph node enlargement & 16 & 21.6 \\
\hline Laryngocele & 2 & 2.7 \\
\hline Lipoma & 19 & 25.7 \\
\hline Ludwig's angina & 1 & 1.4 \\
\hline Lymphangioma & 1 & 1.4 \\
\hline Nerve sheath tumour & 1 & 1.4 \\
\hline Parotid carcinoma & 8 & 10.8 \\
\hline Parotitis & 4 & 5.4 \\
\hline Pleomorphic adenoma & 9 & 10.8 \\
\hline Sebaceous cyst & 6 & 8.1 \\
\hline Submandibular gland abscess & 1 & 1.4 \\
\hline Warthin's tumour & 1 & 1.4 \\
\hline \multicolumn{2}{|c|}{ Total } & 3 \\
\hline Distribution of Clinical Diagnosis of the Swellings \\
\hline \multicolumn{2}{|c}{} \\
\hline
\end{tabular}

\begin{tabular}{|c|c|c|}
\hline FNAC & Frequency & Percent \\
\hline Adenoid cystic carcinoma & 1 & 1.4 \\
\hline Branchial cleft cyst & 1 & 1.4 \\
\hline Laryngocele & 2 & 2.7 \\
\hline Lipoma & 19 & 25.7 \\
\hline Lymphangioma & 1 & 1.4 \\
\hline Nerve sheath tumour & 1 & 1.4 \\
\hline Parapharyngeal abscess & 1 & 1.4 \\
\hline Parotitis & 4 & 5.4 \\
\hline Pleomorphic adenoma & 8 & 10.8 \\
\hline Sebaceous cyst & 6 & 8.1 \\
\hline Squamous cell carcinoma & 26 & 35.1 \\
\hline Submandibular gland carcinoma & 1 & 1.4 \\
\hline Warthin's tumour & 3 & 4.1 \\
\hline Total & 74 & 100.0 \\
\hline
\end{tabular}




\begin{tabular}{|c|c|c|}
\hline $\begin{array}{c}\text { Provisional Diagnosis based on } \\
\text { CT/MRI }\end{array}$ & Frequency & Percent \\
\hline Branchial cleft cyst & 2 & 2.7 \\
\hline Carcinoma buccal mucosa & 1 & 1.4 \\
\hline Carcinoma larynx & 1 & 1.4 \\
\hline Cervical lymph node enlargement & 10 & 13.5 \\
\hline Laryngocele & 2 & 2.7 \\
\hline Lipoma & 22 & 29.7 \\
\hline Lymphangioma & 1 & 1.4 \\
\hline Nerve sheath tumour & 1 & 1.4 \\
\hline Parapharyngeal abscess & 4 & 5.4 \\
\hline Parotid carcinoma & 11 & 14.9 \\
\hline Parotitis & 1 & 1.4 \\
\hline Pleomorphic adenoma & 11 & 14.9 \\
\hline Sebaceous cyst & 3 & 4.1 \\
\hline Submandibular gland carcinoma & 1 & 1.4 \\
\hline Submandibular gland sialolithiasis & 1 & 1.4 \\
\hline Warthin's tumour & 2 & 2.7 \\
\hline Total & 74 & 100.0 \\
\hline \multicolumn{3}{|c|}{ CT/MRI Distribution of Neck Swellings } \\
\hline
\end{tabular}

\section{DISCUSSION}

FNAC has an essential role in the evaluation of patients with non-thyroidal neck swelling. The present study was undertaken to evaluate preoperatively with the help of CT/MRI scan and to correlate the observations with the FNAC examination in order to determine the usefulness of the technique.

In the present study, 23-gauge and one and a half inch long disposable needle was used. Most workers agree that there is nothing to choose in the gauge from 22 - 27 . Friedman et al (1979) ${ }^{6}$ and Braun and Silver (1984) 7 used 18and 19-gauge needles respectively for cystic lesions. But many authors have aspirated fluid with fine needle also. Large needles are traumatic and the aspirate is mixed with blood.

The length of the needle favoured was one and a half inches and in the present study the same was found to be of optimum length. Longer needle bends when it is manipulated within the swelling. All the workers used disposable needles including in the present study, as they are least traumatic, less painful and perfectly sterile.

Most operators employed $10 \mathrm{~mL}$ or $20 \mathrm{~mL}$ syringes. Thomas A Colacchio et al (1980) 8 used $5 \mathrm{~mL}$ syringe. $1 \mathrm{~mL}$ tuberculin syringe with a $1.5 \mathrm{~cm}$ long 25-gauge needle mounted on it was employed by Norton LW et al (1982). ${ }^{9}$ In our series, a $10 \mathrm{~mL}$ disposable syringe was used. Majority of the authors used special syringe holder. Special syringe holder helps to apply suction and release with one hand only, while the other hand fixes the swelling.

A local anaesthetic was infiltrated into the skin to avoid pain to the patient by Colacchio TA et al (1980), ${ }^{8}$ Norton W et al (1982). ${ }^{9}$ None of the patients complained of significant pain in the present study. There is no need of local anaesthesia we feel, when a fine and disposable needle (22$24 \mathrm{G}$ ) is used. Complications after FNAC of non-thyroidal neck swelling were usually not seen. All patients were cooperative.

Majority (74) of the aspirates done by routine FNAC were satisfactory for cytological evaluation with satisfactory smear rate of $87.05 \%$. Afroze et al, Friedman et al and Hyang Miko et al reported satisfactory smear rates in excess of $90 \%$. The rate in our present study $(87.05 \%)$ was better than those in studies by Kamal et al (70.02\% - 72.4\%) and was comparable to studies by Silverman et al ( $87 \%$ - 88.34\%). ${ }^{10,11,12,13,14}$

The age of the patients subjected to FNAC varied from 12 to 73 years with the mean age of 45.62 years. The mean age of the group studied by Rajbhandari M 15 et al was 42 years. Colacchio et $\mathrm{al}^{8}$ reported the age of their patients ranging between 30 and 60 years. In the present series, patients in the $4^{\text {th }}$ and $5^{\text {th }}$ decades of life comprised $44.5 \%$.

The sex ratio (female: male) in the present study group was 1.6: 1 with 46 men and 28 women. In the series of Rajbhandari $\mathrm{M}$ et al, female-to-male ratio was 1: 1.6. Male proportion in the Colacchio et al series was $16 \%$.

In the present study, provisional diagnosis based on CT/MRI correlated with FNAC. Correlation was present in the $61.5 \%$ of non-thyroidal neck swelling.

Pleomorphic adenoma is the most common tumour, which accounts for $60 \%-70 \%$ of all the salivary gland tumours. The reliability of FNAC in diagnosing it has been reported as $90 \%$ - 97\%.16 Minor variations of the cytomorphological features are occasionally seen due to duct cell metaplasia (mucinous, squamous, oxyphilic and sebaceous), variable stromal cellularity, crystal deposition (tyrosine) and intranuclear cytoplasmic inclusions. Among the 74 cases of non-thyroidal neck swellings in the present study, 8 cases (10.8\%) were diagnosed cytologically and 9 (14.9) cases were diagnosed by CT/MRI. About $75 \%$ of pleomorphic adenoma can be correlated with CT/MRI. DWI favours pleomorphic and helps to distinguish it from a Warthin's tumour.

Warthin's tumour is the second most common benign salivary gland tumour $(5 \%-6 \%)$ and a majority of these can occur in the parotid or periparotid area. A combination of oncocytes, lymphoid tissues and cystic macrophages help in the diagnosis. In the present study, $66.7 \%$ correlation was present in case of Warthin's tumour.

Cystic lesions of head and neck can be routinely diagnosed with conventional MRI sequences, but in certain situations such as infected branchial cleft cysts or epidermal cysts, DWI can play an important complementary help.

The malignant tumours spread by the direct and the haematogenous routes. Interestingly, $20 \%$ of the parotid glands, $34 \%$ of the submandibular glands and $14 \%$ of the minor salivary glands will have cervical lymph node metastases at presentation. About 10 cases (13.5\%) were presented with cervical lymph node enlargement. 38.5\% of squamous cell carcinomas were correlated by CT/MRI. 100\% of adenoid cystic carcinoma, nerve sheath tumour and submandibular gland carcinoma were correlated with CT/MRI report. Bryan et al reported that by computed tomography, all the benign lesions were correctly identified as benign, however $17 \%$ of the malignant lesions were incorrectly considered to be benign. ${ }^{17}$

Lipomas constituted as most common benign tumours followed by other benign soft tissue lesions. $89.5 \%$ of lipoma were correlated with CT/MRI. In Pushpender et al case series, CT appearances were diagnostic in patients with cervical lipoma, mesenteric cysticercosis, cervical lymphangioma and thyroglossal duct cyst. Excellent 3-Dimensional imaging is possible using volume rendering maximum intensity projection and shaded surface display techniques, which facilitates the surgeon to understand the anatomical extent of 
the lesion and its relationship to surrounding structures in a much better way. ${ }^{18}$

Other rare swelling which are correlated, 100\% were branchial cleft cyst, laryngocele, lymphangioma, parapharyngeal abscess and parotitis. Ishwar Charan et al found in a case series of 73 patients, sensitivity of CT for tumour extension is more than $82 \%, 10$ evaluation of paraganglioma from other mimicking lesions where multidetector CT (MDCT) sensitivity shows 83.33\%. Diagnostic accuracy of computed tomography in the detection of necrosis in metastatic cervical nodes from patients with head and neck squamous cell carcinoma showed an accuracy, sensitivity and specificity of 92\%, 91\% and $93 \%$, respectively. ${ }^{19}$ Epidermal cysts typically associate restriction in diffusion and low ADC values and are located frequently in the head and neck at first decade. 20

\section{CONCLUSION}

Contrast enhanced CT scans for the neck has improved the localisation and characterisation of neck lesions. Since CT is fast, well tolerated and readily available, it can be used for initial evaluation, extension of the mass, preoperative planning, biopsy targeting and post-operative follow-up. However, FNAC remains the gold standard for making a histological diagnosis. With the advantages of improved vascular contrast enhancement, increased detection of lesions and multiplanar three-dimensional reconstructions multi-slice spiral CT should be one of the modalities of choice in the evaluation of neck masses. DWI is now technically feasible in the head and neck regions. DWI to MRI protocols in the evaluation of head and neck malignancies increases lesion detection and helps the differentiation process between both solid and cystic lesions and benign from malignant lesions. Furthermore, recent series have claimed a role for DWI in primary tumour follow-up, in the evaluation of treatment response and in the detection of local recurrence. Hence, a combination of CT/MRI and FNAC will give comprehensive information regarding the nature of lesion and its extent, which is absolutely necessary for diagnosis and staging of the disease for planning the treatment modality to be adopted.

\section{Limitations of the Study}

The study sample selected is not representative of the general population. So, the true incidence of various non-thyroidal swellings could not be assessed. The number of malignant cases of neck swelling studied during this limited period was not sufficient enough to make concrete conclusions with regard to the sensitivity, specificity and accuracy of CT/MRI.

\section{REFERENCES}

[1] Faizal B, Bhate JJ, Hiran KR. Reliability of fine needle aspiration cytology in salivary neoplasms: surgeon's perspective. Amrita Journal of Medicine 2014;10(2):23-9.

[2] Gupta M, Gupta S, Gupta VB. Correlation of fine needle aspiration cytology with histopathology in the diagnosis of solitary thyroid nodule. J Thyroid Res 2010;2010:379051.
[3] Dipanwita D, Sarma MC, Sharma A, et al. A comparative study between fine needle aspiration cytology and histopathological examination in the diagnosis of neoplastic and nonneoplastic lesions of the thyroid gland. Indian J Prev Soc Med 2012;43(1).

[4] Suen KC, Quenville NF. Fine needle aspiration biopsy of the thyroid gland: a study of 304 cases. J Clin Pathol 1983;36:1036-45.

[5] Walfish PG, Hazani E, Strawbridge HT. Combined ultrasound and needle aspiration cytology in the assessment and management of neck swelling. Ann Intern Med 1976;143:365-8.

[6] Friedman M, Shimaoka K, Rao U. Diagnosis of chronic lymphocytic nodular presentation of neck mass by needle aspiration. Acta Cytol 1981;25:513-22.

[7] Braun RJ, Silver CE. Needle aspiration biopsy of the neck mass. Laryngoscope 1984;94:38-42.

[8] Colacchio TA, LoGerfo P, Feind CR. Fine needle aspiration diagnosis of thyroid nodules. Review and report of 300 cases. Am J Surg 1980;140(4):568-71.

[9] Norton LW, Wangensteen SL, Davis JR, et al. Utility of aspiration biopsy. Surgery 1982;92(4):700-5.

[10] Silverman JF, West RL, Larkin EW, et al. The role of fine-needle aspiration biopsy in the rapid diagnosis and management of thyroid neoplasm. Cancer 1986;57(6):1164-70.

[11] Afroze N, Kayani N, Hasan SH. Role of fine needle aspiration cytology in the diagnosis of palpable thyroid lesions. Indian J Pathol Microbiol 2002;45(3):241-6.

[12] Kamal MM, Arjune DG, Kulkarni HR. Comparative study of fine needle aspiration and fine needle capillary sampling of thyroid lesions. Acta Cytol 2002;46(1):30-4.

[13] Friedman M, Shimaoka K, Getaz P. Needle aspiration of 310 thyroid lesions. Acta Cytol 1979;23(3):194-203.

[14] Ko H, Jhu IK, Yang SH, et al. Clinico pathological analysis of FNAC of the neck leision. Acta Cytologica 2003;47:727-32.

[15] Rajbhandari M, Dhakal P, Shrestha S, et al. The correlation between fine needle aspiration cytology and histopathology of head and neck lesions in kathmandu university hospital. Kathmandu Univ Med J (KUMJ) 2013;11(44):296-9.

[16] Layfield LJ, Tan P, Glasgow BJ. Fine-needle aspiration of salivary gland lesions. Comparison with frozen sections and histological findings. Arch Pathol Lab Med 1987;111(4):346-53.

[17] Bryan RN, Miller RH, Ferreyro RI, et al. Computed tomography of major salivary glands. Am J Roentgenol 1982;139(3):547-54.

[18] Gupta P, Bhargava SK, Mehrotra G, et al. Role of multi slice spiral C.T. in the evaluation of neck masses. JIMSA 2013;26(1):51.

[19] Charan I, Kapoor A, Kumar N, et al. Evaluation of neck mass with computed tomography: an observational study. International Journal of Scientific Study 2014;2(7):118-22.

[20] Barbero JPM, Jiménez IR, Noguerol TM, et al. Utility of MRI diffusion techniques in the evaluation of tumors of the head and neck. Cancers 2013;5(3):875-89. 